\title{
LA CRISIS DEL REFORMISMO EN CAMPOMANES
}

\author{
Antonio ALVAREZ DE MORALES \\ Universidad Autónoma de Madrid
}

Quizá ninguna figura como Campomanes resume tan bien la crisis del reformismo ilustrado y refleja sus contradicciones de forma tan clara.

Campomanes expresa mejor que ningún otro personaje político de la segunda mitad del siglo XVIII las características de este reformismo que el rey Carlos III no dudó en alentar en todo momento.

Tuvo la suerte de pertenecer desde el primer momento al grupo social que el rey favoreció y que por consiguiente acabó haciéndose con el poder en detrimento de la llamada casta colegial que lo había detentado anteriormente. Conscientes todos ellos de que su acceso al poder se lo debían exclusivamente al rey no dudaron en exaltar hasta el paroxismo el poder real.

Es evidente que en los países católicos como España, la Iglesia Católica constituía una limitación de ese poder real, de aquí la trascendencia que podía tener la difusión del regalismo. A pesar de que esta corriente ideológica estaba presente en el seno de la Monarquía española desde sus orígenes en la Edad Media, no fue precisamente en España donde se desarrolló más, y quedó siempre con una significación y un alcance muy limitado '. El siglo XVIII propició en toda Europa una mayor difusión de las doctrinas regalistas favorecida sin duda por la decadencia del poder político de los Papas, condicionado como nunca por los monarcas europeos. No es, por consiguiente, extraño que en España también se difundiera con mayor ahínco la doctrina regalista. Pero en general tanto la historiografía del talante conservador que ha difundido una imagen de los políticos de Carlos III marcada por la expulsión de los jesuitas, dándole a esta medida una significación religiosa que no tuvo, como otras corrientes historiográficas no tan conservadoras, o que incluso se han pretendido poner en frente de éste, nos han dado una imagen regalista de la política seguida por este grupo de políticos que acceden al poder en el reinado de Carlos III, pero podemos ver aquí de una forma clara la debilidad de este regalismo y de las contradicciones de este reformismo.

Es cierto que en los escritos de Campomanes, sobre todo en los de sus primeros

1 Vid. mi trabajo "La influencia del regalismo en la configuración jurídica de la Inquisición", en Revista de Derecho Público, 108-109 (1987), pp. 731 y ss. 
años de fiscal, se puede encontrar un tono claramente radical en la defensa de las regalías de la corona y precisamente este tono pienso que fue el culpable del estancamiento de su carrera política, mientras que el tono más político de su compañero Moñino facilitaría en cambio la carrera de éste, lo que ya es un índice de las limitaciones del regalismo español de la época.

Si leemos con atención, por ejemplo, el dictamen fiscal sobre la censura de libros que realizaba la Inquisición, veremos cómo se fue desarrollando esta línea reformista hasta su crisis final ${ }^{2}$.

Como es sabido el celo de Carlos III quedó profundamente alterado a comienzos del reinado, en 1762, como consecuencia de la condena por la Inquisición española del libro de Mesenguy, Exposición de la doctrina Cristiana, por su contenido jansenizante. Carlos III se vio sorprendido por la resistencia del Inquisidor General a rectificar esta decisión, lo que provocó un primer encontronazo entre el rey y la Iglesia, que llevó a aquél a autorizar una Cédula Real por la que se establecía una institución jurídica de claros resabios regalistas el "regium exequatur", por la que se sometían a la autorización previa del soberano las bulas y levas pontificias, así como las medidas tanto de la Inquisición romana como de la española.

La aplicación de esta Cédula fue, sin embargo, suspendida a los pocos meses a instancias del confesor del rey, y todo quedó como estaba.

Pero en 1768, tras la expulsión de los jesuitas y la llegada del nuevo equipo gobernante al poder, éste consideró que había llegado el momento de reanudar esta política. El Consejo de Castilla volvió así a estudiar el asunto del Indice de los Libros prohibidos de la Inquisición. Iniciativa que se ha atribuido al nuevo Presidente del Consejo el Conde de Aranda, aunque hoy sabemos mejor las limitaciones en que se desarrolló su acción política en aquellos años. Lo cierto es que el Consejo estudió los medios de mejorar la llamada censura inquisitorial. No es el caso estudiar en detalle esta cuestión, lo que interesa es constatar cómo esta medida comportaba por sí sola una limitación importante del poder que hasta entonces había tenido la Inquisición. De nuevo el Inquisidor General se enfrentó como seis años antes a la medida real, defendiendo el poder de la Inquisición.

El escrito de éste pasó a los fiscales del Consejo para que lo contestaran, y en efecto éstos, como si estuvieran esperando la ocasión, redactaron un largo dictamen defendiendo las regalías de la Corona que fue aprobado por el pleno del Consejo. Cualquiera que lo lea se puede dar cuenta de la base regalista del escrito, el Rey como patrón fundador y "dotador" económico de la Inquisición posee sobre ella los derechos inherentes al patronato real, en tanto que príncipe liberal que ha enriquecido la Inquisición con el ejercicio de la jurisdicción real, su Majestad dispone de forma imprescriptible del derecho de velar por el uso que se hace de esta jurisdicción, de iluminarla y dirigirla, de reformar sus abusos, de limitarla e incluso de suprimirla si la necesidad y la utilidad pública lo pedían. Al margen de los rumores que la publicidad de este dictamen dio lugar no sólo en España, sino en Europa, se ha destacado suficientemente la importancia de este documento como expresión clara del reformis-

2 Consulta del Consejo y resolución de su Magestad a súplica del Inquisidor General sobre las reglas dadas por S.M. para la expurgación y prohibición de los libros, con fecha de 28 de febrero de 1768 . Se trata de un documento no paginado de unas 200 páginas conservado en la Biblioteca Nacional, Ms. 10.863 , apenas utilizado por los historiadores. 
mo ilustrado. Pero importancia nada más que teórica por no decir retórica. Es evidente, que si estos principios se hubieran aplicado, una reforma profunda se hubiese iniciado, en una institución tan significativa como la Inquisición. Sin embargo nada de ello se llevó a cabo, la cédula como tantas disposiciones legales, en todos los tiempos y bajo distintas situaciones políticas, quedó sin aplicación. Pero no sólo esto marca los límites del reformismo y regalismo sino toda la actuación posterior de Campomanes referida muy especialmente a sus relaciones posteriores con la Inquisición, especialmente su actitud ante el proceso de Olavide donde tuvo una actitud tan cobarde.

Refleja también adecuadamente sus limitaciones reformistas su idea sobre la reforma de la justicia, sobre todo si la ponemos en contraste con la reforma que se planteó contemporáneamente en algunas de las Monarquías Absolutas europeas, como es el caso de Prusia.

La reforma de la justicia, que Federico II quería llevar a cabo en Prusia desde su llegada al poder, debía ser realizada de un lado por la vigilancia más estricta de los jueces, tanto por una disciplina más rígida como por leyes mejores.

El rey y los legisladores tenían el mismo objetivo, ligar más fuertemente al juez a los textos legales, solamente el rey buscaba garantizar su derecho al poder mientras que los legisladores inspirados en las ideas de Montesquieu y Beccaria, perseguían la libertad del ciudadano por medio de leyes claras y comprensibles a todos y se oponían a la creación del derecho por los jueces.

El modelo teórico de los legisladores consistía en un absolutismo de las leyes según el cual todo el sistema jurídico debía ser presentado bajo forma escrita y bajo forma de ley. En cambio, el derecho consuetudinario, la interpretación por los jueces o la ciencia iban a ser reemplazados por la ley formal. Las imperfecciones de la ley no podían ser compensadas más que por el monopolio de interpretación de la comisión jurídica.

La concepción ideal de los legisladores de la ley no podía ser transferida más que parcialmente del proyecto de código general al Código civil general (Allgemeines Landrecht). En la práctica el ideal funcionaba bien a causa de la técnica permanente de la creación del derecho no formal que ejercía el monarca, como a causa del desarrollo ulterior judicial del derecho, sobre todo en el campo de la constitución agraria que tenía necesidad de reformas, Savigny rompió con el ideal del Siglo de las Luces poniendo al mismo nivel de la ley como fuente del derecho, el derecho consuetudinario y la ley interpretada por la ciencia. Era como decir que el juez no estaba sometido a la Ley.

Vamos a examinar ahora algunas de las ideas reformistas sobre la justicia de Campomanes ${ }^{3}$.

\section{LA CRITICA DE LOS JUECES Y DEL ARBITRIO JUDICIAL}

La conciencia de la grave crisis de la organización judicial permitía la acción del gobierno sobre la justicia y los jueces, y el debate se centró en el tema fundamental del arbitrio judicial.

3 Todas estas ideas de Campomanes en una carta a D. Manuel de Roda, de 10 de septiembre de 1750, enviándole un ensayo sobre la reforma del Derecho en Archivo Campomanes sign., 60-4, Fundación Universitaria Española, Madrid. 
El amplio ámbito de la discrecionalidad judicial, aunque tiene una cierta elaboración doctrinal en el derecho común tardío, en realidad, se plantea como una situación de hecho que se agravó sobre todo a partir del siglo XVI en que cayó en completo desuso la obligación de motivar las sentencias ante la imposibilidad de motivarlas en base al derecho escrito ante el caos en que éste se encontraba; también ante la dificultad de motivar, basándose en el variopinto e inestable derecho jurisprudencial o en los varios e informales estilos interpretativos y forenses, para no dar base a la oposición de la parte derrotada en la litis y por la inoportunidad para los magistrados de dar a conocer al pueblo las armas de la soberanía que sólo por vías internas el Rey había en cada momento de conocer. Además, en los órganos colegiados el voto de cada uno de los miembros podía estar fundamentado en motivos distintos, aunque se basasen en la misma disposición. Se discutía también si debía prevalecer el criterio de la mayoría absoluta o relativa.

Por otra parte, los gobiernos mostraban un apoyo a esta libertad del juez intérprete, ya que favorecía el despotismo desde la influencia del ministro y se esperaba que la simplicidad facilitara la rapidez. Así, la certidumbre y simplificación del proceso aparecen en esta época movidas por influencias contradictorias.

Aunque aquí Campomanes se muestra radicalmente opuesto al arbitrio judicial, debemos tener en cuenta que ésta no era todavía una opinión mayoritariamente clara en el mundo del derecho español; es de destacar que había corrientes jurídicas que seguían patrocinando el arbitrio judicial, apoyándose en las construcciones sobre la equidad y epiqueya. Así, por ejemplo, por los mismos años en que Campomanes redactaba este escrito, se publicaba por el licenciado Juan Antonio Pardo y Ripa, abogado de Pamplona, un Tractatus de Juris Epiqueya seu Aequanimitatae, Pampilonae, 1751 , en el que se defiende el arbitrio judicial ${ }^{4}$.

Ante el arbitrio de interpretar las leyes los jueces, se lamenta de que el Príncipe, a quien está reservado aclarar las ambigüedades legales, no lo haya hecho así, por lo que los juristas se han hecho "casi despóticos árbitros del Derecho y no fieles depositarios e ingenuos aclaradores de él como debieran".

Campomanes criticaba la mala preparación previa de los jueces, pues no conocían las leyes del Reino y así muchas de las confusiones provienen de mezclar el derecho de España con el romano por no tener conocimiento del primero.

Eligen, además, mal los asuntos por los que se guían y no examinan los procesos por sí mismos, con lo que dan sentencias que no se acomodan a la controversia.

El arbitrio judicial, que es la madrasta de la Justicia, a título de aplicar lo que llaman "graciables" les lleva a un abuso insoportable.

Dice textualmente Campomanes: "Los jueces, que también se incluyen en la clase de profesores de la Jurisprudencia, suelen incurrir en el abuso de ella, pero sus defectos, aunque más perjudiciales al bien público, son más desconocidos, porque tales determinaciones las forman a sus solas, sin necesidad de expresar los motivos en que las fundan por estarles prohibido por las Leyes del Reino justamente por obviar cavilaciones" 5 .

Campomanes toca aquí un punto interesante y sobre el que se había producido

4 Carta citada.

5 Ibíd. 
una situación distinta en los diversos territorios que formaban la Monarquía, pues mientras en Aragón, Mallorca y Cataluña el deber de motivar las sentencias estaba expresamente establecido desde el siglo XVI, en cambio en Castilla este deber no se estableció nunca, y aunque en la Edad Media se tenía costumbre de hacerlo, en la Edad Moderna se desterró esta práctica ${ }^{6}$. Los Decretos de Nueva Planta, aunque plantearon algunas dudas al respecto, no alteraron la cuestión, pues se permitió que las sentencias de las Audiencias afectadas por los Decretos siguieran observando la práctica de motivar las sentencias. Pero en 1778 Carlos III prohíbe motivar las sentencias terminantemente en todos sus reinos, para evitar los perjuicios que resultan con la práctica, que observa la Audiencia de Mallorca, de motivar sus sentencias, dando lugar a "cabilaciones" de los litigantes, consumiendo mucho tiempo en la extensión de las sentencias, que viene a ser un resumen del proceso, y las costas que a las partes se siguen; cuando cese en dicha práctica de motivar sus sentencias, ateniéndose a las palabras decisorias, como se observa en él, "mi Consejo y en la mayor parte de los Tribunales del Reyno; y que a exemplo de lo prevenido a la Audiencia de Mallorca, los Tribunales ordinarios, incluso los privilegiados excusen motivar las sentencias como hasta aquí, con los vistos y atentos en que se refería el hecho de los autos y los fundamentos alegados por las partes; derogando, como en esta parte derogo, el auto acordado 22, título 2, lib. 3, duda 1, de la Recopilación, u otra cualquiera resolución o estilo que haya en contrario" 7 .

Finalmente, señala que la diferencia entre el cohecho y el empeño es que en el primer caso la justicia se vende al contado y en el segundo al fiado, pero el resultado es el mismo.

Critica así mismo la tardanza y la ambigüedad de las providencias que adoptaban los tribunales.

\section{LA REFORMA DE LOS ESTUDIOS DE JURISPRUDENCIA}

En el capítulo tercero, "Modo de dirigir los estudios de jurisprudencia en España" trata ya de poner los remedios a la situación empezando por plan de estudios nuevo para las Facultades de Leyes. Propone que se estudiará Retórica y Didáctica,

6 En España era distinta la situación según se trataran de tribunales del Reino de Castilla o de los territorios de la Corona de Aragón; en estos últimos, desde 1547, existía la obligación de los jueces de entregar su voto con los motivos del mismo y estos votos eran registrados en el libro existente para ello: "...todos los Consejeros y Asesores que han de aconsejar, y los Jueces que han de juzgar sean obligados, al tiempo que votaren, decir las causas y fundamentos principales, así de Fuero como de Derecho, así en lo civil como en lo criminal por donde se mueven a dar sus votos: los cuales se hayan de asentar en el libro de consejo de tal Juez...". Fueros y Observancias del Reino de Aragón, Zaragoza, 1624, Lib. VII, f. 133.

En Castilla, el Libro Secreto, su Acuerdo (Ordenanza de Medina del Campo de 1489, recogida en la Novísima, Libro V, Titulo I, Ley XI) rompía con la tradición establecida desde Las Partidas (Tercera Partida, título XXII, ley XVIII) que admitía la publicidad del voto contrario. En el Libro Secreto pasaban a recogerse sin publicidad estos votos, pero sin motivar. El art. 367 de la L.E.C. de 1891, todavía vigente, recogió, al menos teóricamente, la tradición del Libro Secreto, previendo que el voto sea recogido sin manifestarlo en la sentencia, pero que sea comunicado al Tribunal que entienda de la apelación de esa sentencia.

7 Novísima Recopilación, 11, 16, 8. R. Cédula de Carlos III de 23 de junio de 1778. 
pero sin "las quisquillas metafhisicas que nos sirven para hacer los hombres sofísticos y charlatanes que no para formarlos juiciosos, penetrantes e ingenuos".

Para el Estudio Legal Teórico: Instituta de Justiniano directamente sin el Vinnio, pues era demasiado obscuro; Pichardo era más claro, pero tampoco lo recomendaba; Harpecto era amplísimo y no servía para principiantes.

Recomendaba luego algo de Pandectas y de Historia del Derecho Romano por Gravino, Heineccio o Brunkel.

Pero había que estudiar Derecho español, sobre todo la Nueva Recopilación y las Partidas, pero también lo que llamaba Fuentes del Derecho Español: Fuero Juzgo, Fuero Real, ya que muchas de las disposiciones estaban vigentes, y el derecho feudal también se debía estudiar; además del Derecho público: Derecho internacional, Derecho marítimo, Derecho tributario. Pero faltaba un libro adecuado.

\section{ESTUDIO LEGAL PRACTICO}

El estudio legal práctico era fácil para Campomanes, siempre que el estudio teórico se hubiera hecho bien. La práctica se debía aprender de "viva voz" en los despachos profesionales y en las academias. La actividad de pasante en un despacho de abogado había sido sistema habitual desde hacía tiempo; lo que constituía cierta novedad eran las academias que se habían comenzado a formar en Madrid en el siglo XVIII, precisamente dirigidas a la preparación práctica de los abogados además en Madrid, en donde estaban los principales Tribunales del Reino, pero carecía de Universidad.

Este estudio práctico se debía completar con la Curia Filípica y las Manuducciones de Pichardo. Además, se debía tener una Biblioteca jurídica como la que emprendió Lucas Cortés, y mientras esto se hacía podían servir las de Draudio, Lipeni, Struvio, Simón Morhof ${ }^{8}$ y la Themis Hispana de Franckenau, así como las de Nicolás Antonio, Rodríguez y Ximeno y la de Barbosa.

Para el derecho canónico especialmente se debían tener en cuenta las colecciones de los Concilios, como las de Carranza y Aguirre.

\section{LA REFORMA DEL DERECHO PROCESAL}

La situación de la administración de justicia en el siglo XVIII era lamentable; a

8 De Martin Lipeni es Bibliotheca realis juridica post V.V.C.C. Fried. Gotl. Struvii et Gottl. Jenicchenii iuris emendata, multis accesionibus aucta et locupletata, adject etiam accuratto scriptorum. Indice instructa, en 2 vols. Lipsiae (Leipzig), 1757. La edición que manejó Campomanes debió de ser una anterior.

En 1775 Schott publicó una nueva edición con adiciones suyas. En 1789 el barón de Senckenberg hizo lo mismo. Todavía a principios del siglo XIX tuvo dos ediciones más.

B. G. Struve, autor de Biblitoheca juris selecta secundum ordinem litterar, disposita et ad singulas juris partes directa, accesit Bibliotheca selectissima juris estudiosorum, emendar. et copiose locuplet. Christi. Gli Buster Ed. VIII Jenae (703). Se publicaron suplementos.

El alemán Jorge Draud, latinizado Draudio (1573-1630) es famoso por su Bibliotheca librorum germanorum classica, Francfort, 1625, que es la obra a que se refiere Campomanes.

Simón Morhof (1639-1691) es también un literato alemán famoso, sobre todo por su Polyhistor, nombre que se daba a las enciclopedias literarias, editada por primera vez en Luibeck, 1688, y reeditada por cuarta vez en 1744. 
ello habían contribuido razones de fondo y abusos más o menos importantes, pero que, todos juntos, ofrecían un aspecto desolador de la justicia. No es de extrañar, por consiguiente, que surgieran deseos de reforma que se llegaran a plasmar incluso en propuestas concretas como ésta que comentamos de Campomanes, escrita cuando aún era joven abogado y titulada de forma significativa "Método de acortar los pleitos y los abusos que les hacen interminables".

\section{JUICIO ORDINARIO}

Era el más dilatado de todos. Campomanes propone terminar con el arbitrio de los jueces en la concesión de prórrogas de los plazos, que conculcaban todas las leyes de la Recopilación dirigidas a acortar los pleitos. También propone terminar con los artículos de previo y especial pronunciamiento, que debían limitarse sólo si se referían a las cuestiones siguientes: dedicatoria de jurisdicción, acumulación o separación de autos, o de no contestar. Igualmente se debía prohibir la posibilidad, admitida por los prácticos, de solicitar al juez que reforme los autos por contrario imperio. Lo mismo sucede con las nulidades que, después de pronunciadas las sentencias, podían pedir las partes dentro de los 60 días inmediatos a ella, dejando a salvo el derecho de la apelación ", se suprimirían también las demandas de "jactancia" 10 y la prueba de tachas ${ }^{11}$, ya que no daban lugar más que a alargar indefinidamente los juicios.

9 La Ley 12, título 22, partida 3 recogida en la Ley 2, título 16, libro 11 de la Novísima Recopilación, permitía plantear alegación de nulidad ante el mismo juez que la había pronunciado o en la apelación, incluyendo la cláusula salvo el derecho de nulidad en los siguientes casos: si el juez no tiene jurisdicción o es incompetente por cualquier razón, cuando la sentencia no contiene absolución o condenación en todo o en parte, o no designa la cosa o cantidad de que absuelve o condena, cuando el juez la da fuera del lugar acostumbrado o no la hace escribir o la pronuncia sin emplazar u of́r a la parte o sin estar contestada la demanda o si no cita a las partes para que asistan a oírla, cuando se da contra el que debiendo tener acusador no lo tuviese salvo que le fuese favorable, cuando es contraria a las leyes, a la naturaleza o a las buenas costumbres, cuando se dé un día feriado o de noche o en cosas espirituales por juez lego, cuando se pronuncia contra la autoridad de la cosa juzgada, cuando se prueba que el juez la dio por dinero, cuando no fuere conforme a la demanda.

10 Los juicios de jactancia, regulados por la Ley 46, Título 2, Partida 3, son aquellos que se podían iniciar por una manifestación que alguien hacía contra otro de cosas que podían causarle algún perjuicio en su estado personal o reputación. En estos casos el agravio podía exigir al juez que obligase al jactancioso a poner demanda para probar sus manifestaciones, a desdecirse judicialmente de ellas $o$ a darle la satisfacción que corresponda.

La acción de jactancia viene ya recogida en las Flores del Derecho Libro I, título XIV, ley 2, pp. 90 92, ed. de Ureña y Bonilla. Vid. SENTIS MELENDO: "El juicio de jactancia (ensayo de sistematización bibliográfica y jurisprudencial)", en Revista de Derecho Procesal, II (1943), pp. 113-172.

11 La prueba de tachas, según la Ley 1, título 12, libro II de la Novísima Recopilación, era realizada a los testigos que presentaban las partes del juicio; las tachas eran los defectos que alegaban las partes ante el juez contra los testigos para impedir que éste diese crédito a sus declaraciones. Las tachas podían recaer sobre la propia persona del testigo y sobre sus declaraciones o sobre su examen. Para que se admitiesen las tachas éstas debían ser especiales, es decir, provocadas por un hecho concreto; esto es, si por ejemplo se tachaba a un testigo de falsario, había que alegar el hecho concreto de que había sido falsario. Legalmente las tachas debían proponerse dentro de los seis días siguientes al de la notificación por probanzas, pero los autores que trataron ampliamente de este tema consideraban que este plazo no podía correr mientras las partes no hubiesen oído las declaraciones de los testigos, y por influencia de la doctrina de los jueces entendían con gran flexibilidad este plazo, con lo que este trámite se alargaba de forma impredecible. 
La reducción de las jurisdicciones exclusivamente a la real, la espiritual y la militar facilitaría la reducción de pleito, pues no había pleito que no se iniciara con un conflicto de competencias como consecuencia de este exceso de jurisdicciones.

Campomanes señalaba también la necesidad de suprimir determinados procesos especiales, como los juicios posesorios separados de los de propiedad, las restitutio in integrum; los pleitos de comunidad de pastos para terminar los cuales proponía no el amojonamiento, que era insuficiente para evitarlos, sino una normativa general a la que todos se viesen; las causas de la Mesta provocadas ya sólo por el abuso de los Alcaldes Mayores entregadores que cobraban en función de los pleitos que tuvieran. Para los rompimientos de dehesas o adehesamiento de tierras que causaban muchos litigios, proponía como solución que se hiciera una lista general de las dehesas del Reino, deslindándolas y señalando su cabida y terreno de calidad, entendiendo que las que no estuvieran en esta lista eran de mala calidad y por tanto se redujeran a pastos y labor. Supresión del derecho llamado de tiras que se pagaba en las escribanías para tomar a las partes el pleito que iba en apelación al Tribunal Supremo y se regulaba por las hojas, pagando un tanto por cada una.

Especialmente significativa es la crítica que hace a las elecciones de justicias en los pueblos, sobre todo por el mito histórico que sobre esta cuestión levantó cierta historiografía. Las elecciones eran causa de infinidad de pleitos y "el agotadero de la subsistencia de los pobres", que surgían de la ambición de mandar y de la diversidad del sistema por el que se llevaban a cabo esas elecciones. La reforma que Campomanes proponía en este punto era radical, terminar con las elecciones y que se diese una fórmula uniforme de nombrar los justicias, reduciendo todo el territorio a partidos ${ }^{12}$.

El que las alegaba debía jurar que no les ponía las tachas por malicia ni con afán de calumniar. Las tachas se podían poner en interrogatorio o en pedimento y el juez, si las admitía, debía abrir un período probatorio (vid. Ley 1 y 2, título 12, libro II, Novísima Recopilación, y Ley 32, título 16, Partida 3, Capítulo 31, De testibus).

12 Dice literalmente Campomanes en su Informe: "Las elecciones de oficios de Justicia en los pueblos es la piedra de escándalo que causan bandos y ojerizas en los vecindarios. Son víctimas de infinidad de pleitos y el agotadero de la subsistencia de los pobres: ésta nace de la ambición de mandar y de la variedad que observan los pueblos en las elecciones, y si bien esto puede remediarse, la calificación de las personas siempre es materia que pide conocimiento de causa. Yo creo no ser remediable este mal, si no se quita el derecho de elegir a los pueblos, dándose una forma constante de proveer justicias o bien reduciéndolo todo a partidos y poniendo en éstos jueces a propósito o tomando otras medidas que parezcan a el Gobierno más oportunas".

La medida que finalmente se adoptó fue la propuesta por Campomanes en circular de 31 de marzo de 1761. "Teniendo presente el Consejo los inconvenientes, perjuicios y perturbaciones que se seguían de que en los pueblos se hiciesen las elecciones en este asunto, la uniformidad para evitar las reiteradas quejas y recursos a que daban causa muchos Alcaldes y Regidores del Reino, por mantenerse en el manjeo con el pretexto de no tener hecha la cobranza de reales contribuciones y otros fines particulares en daño del bien común y para ocurrir a él con remedio oportuno se manda que en día primero de cada año, incluso el siguiente de 1762 , se lleven a efecto todas las elecciones correspondientes a él, que no se contradijeron por excepciones legales que padezcan así en los pueblos del Realengo, como en el Señorío y Abolengo y en las que precede proposición la hagan con un mes de anticipación...".

Vid. Colección de Pragmáticas, Cédulas... del Señor D. Carlos III, por D. Santos Sánchez, Madrid 1803, p. 20. 


\section{EL JUICIO EJECUTIVO}

Especial atención presta al juicio ejecutivo y a los abusos que en él se daban y que se concretaban sobre todo en el abuso de la décima que iba en beneficio de los jueces y no de los acreedores como estaba previsto, lo que perjudicaba a los deudores, ya que esta multa se estableció como pena a la tardanza de los deudores morosos, que dentro de las doce horas de despacharse la ejecución no pagasen las deudas.

El mismo abuso se había producido con el plazo de diez días que establecía la ley para que el acusado probase las excepciones de quita o paga, pacto de no pedir u otros semejantes, ya que al ser un plazo legal el juez no debía ampliarlo por causa alguna.

Una situación similar se producía en los juicios ejecutivos que se referían a los atrasos de censos por los caídos de nueve años y medio, en que se obligaba al censualista a llevar también la reclamación por vía ordinaria, provocando dos pleitos sobre el mismo asunto.

Las oposiciones o tercias dotales, la cesión de bienes y la concurrencia de acreedores eran los expedientes a los que se acudía habitualmente para entorpecer los juicios ejecutivos.

En este apartado también incluía Campomanes las testamentarias y abintestatos, pues los consideraba asimilables a los juicios ejecutivos, denunciando los abusos de los contadores que alargaban indefinidamente su trabajo con el único fin de cobrar más, ya que lo hacían en función de su extensión.

Campomanes proponía que se redujeran a sumarios los pleitos de tanteos o retractos legales y gentilicios.

Abordaba a continuación el problema de las sentencias arbitrales con la fianza prevenida por la llamada Ley de Madrid, que se había convertido en una "sentina de litigios", por los recursos a que habitualmente daban lugar eran "bien intrincados y casi interminables" 13 .

Finalmente, los remates y pregones para la venta y subasta de bienes estaban llenos de mil inútiles formalidades que proponía suprimir sustancialmente.

\section{JUICIO CRIMINAL}

Campomanes hacía las mismas consideraciones que antes había hecho sobre los otros tipos de juicio, sobre la necesidad de agilizarlos, pero aquí poniendo mayor énfasis por los mayores perjuicios que en este tipo de juicio se podía causar a los afectados.

\section{APELACIONES Y REVISTAS}

Para las apelaciones, Campomanes aplicaba la filosofía general de que está inmerso su escrito, es decir, reducirlas a lo estrictamente necesario.

13 La fianza de la Ley de Madrid era la que había que prestar en los juicios ejecutivos que dimanaban de sentencias arbitrales, transacciones y juicios de contadores y tenía por objeto la restitución de lo cobrado con los frutos y rentas. Se llamaba de Madrid por haber sido establecida por los Reyes Isabel y Fernando en las Ordenanzas de Madrid de 1502. Leyes 4 y 5, título 17, libro II, Novísima Recopilación. 
Las revistas eran las apelaciones ante las sentencias de los tribunales superiores, proponiendo, para reducirlas drásticamente, que si además de la sentencia del Tribunal superior hubiese ya otra inferior, sólo se admitiese en este caso el pedimento de súplica o prestación de nuevo instrumento.

\section{JUICIOS EXTRAORDINARIOS}

Se ocupaba bajo este apartado, que rotulaba como juicios extraordinarios, de los juicios de tenuta, recursos de fuerza y retención y el recurso de segunda suplicación, en los que advertía que no sería "justo innovar" ya que respondían a los criterios que trataba de defender.

\section{PLEITOS ECLESIASTICOS}

El furibundo regalista que pareció ser Campomanes se mostraba prudente en este apartado bien significativo, adelantando que aquí procedía por vía de humilde representación al Pontífice para que de acuerdo con el Monarca procediesen ambos a superar los defectos existentes.

Sus propuestas, sin embargo, eran las siguientes: la principal, que se suprimiese el requisito de las tres sentencias que hacía interminables los pleitos, provocaba grandes gastos, sobre todo al tener que acudir a Roma, y producía grandes agravios por la ignorancia que lógicamente tenían de las costumbres del Reino los Tribunales de la Curia Romana. Para resolver estos problemas proponía que la tercera sentencia se dictase siempre en España ante el Tribunal de la Nunciatura ${ }^{14}$.

A continuación abordaba el tema de los pleitos matrimoniales y el abuso de que con sólo los esponsales ligeramente justificados se procediese a captura, cuando además éste era un juicio puramente civil. Campomanes, al proponer remedios para esta cuestión, entraba casi en el terreno del moralista, señalando que por sí ni los esponsales ni el estupro dieron lugar al matrimonio; las mujeres serían más cautas y los matrimonios se celebrarían por medios más honestos.

Igualmente el abuso de los pleitos de divorcios y nulidad de matrimonios constituían un grave escándalo, por lo que Campomanes proponía que el causante de un divorcio sufriera una penitencia austera y que, en todo caso, durante el juicio, bien de divorcio, bien de nulidad, los conyuges deberían, separados, seguir unos ejercicios

14 El Tribunal de la Rota fue creado en 1771 , y en ello intervino decisivamente Roda, el personaje a quien Campomanes envía este escrito. Existía desde el siglo XVI un Tribunal de la Nunciatura Apostólica en España que entendía en primera instancia tanto de causas civiles y criminales que afectasen a los regulares y demás personas con fuero eclesiástico y también actuaba como tribunales de apelación en sentencias dadas por los obispos españoles. Hubo muchos problemas y los regalistas se enfrentaron a él; por ello se negoció con Roma en varias ocasiones para conseguir su supresión y, por fin, se consiguió en 1771 estableciendo en su lugar el Tribunal de la Rota inspirado en el de Roma, y precisamente en la línea que aquí exponía veinte años Campomanes, es decir, como Tribunal de apelación que evitase llevar los pleitos a Roma. Vid. VALES FAILDE, J.: La Rota Española, Real Academia de Ciencias Morales y Políticas, Madrid, 1920; y CANTERO, Pedro: La Rota Española, C.S.I.C. Madrid, 1946. 
espirituales a cargo de sus párrocos. Lamentables, sobre todo, eran para él las nulidades por impotencia que daban lugar a "sucios procesos".

En un plano más regalista, critica el exceso de los eclesiásticos en el uso de la excomunión recordando que el Concilio de Trento había ordenado que su utilización fuera lo más parca posible. Esto le daba pie para entrar en uno de los temas más queridos a los regalistas, las censuras de la Bula de la Cena, negando su validez en España ${ }^{15}$, recordando los argumentos utilizados por el fiscal José Ledesma. Como es sabido, las críticas a esta Bula arreciaron de tal manera en el siglo XVIII, que los Papas acabaron por renunciar a ellas.

Los beneficios eclesiásticos y las capellanías de sangre daban lugar a infinidad de litigios por el afán de los eclesiásticos por pleitear y por la manía de los fundadores de establecer fundaciones complicadas.

El antiforalismo de Campomanes se manifestaba también aquí en un pequeño detalle que afectaba sobre todo a Aragón y Cataluña en donde los pleitos eclesiásticos se actuaban en latín y con mil rodeos forales, causando gran confusión cuando se apelaban. Campomanes proponía perfecta uniformidad en los tribunales de todo el Reino y que sólo se actuase en "español y en igual armonía".

$\mathrm{Ni}$ que decir tiene que casi nada de lo que propone reformar Campomanes se reformó. En definitiva, como ocurrió también en otros países, el Reformismo Ilustrado, especialmente cuando afectaba a cuestiones delicadas, prefirió mantener una actitud pasiva.

15 Censura de la Bula de la Cena. La Bula In Coena Domini era una constitución pontificia que se promulgaba solemnemente en Roma los Jueves Santos y donde se publicaban unas excomuniones contra una serie de delitos. Se llamaba también Processus o Litterae Processus y su origen está en los procesos de excomunión que en Roma se hacían desde el siglo XIII, que quedaron reservados a la absolución del Romano Pontífice. A partir de la Bula dada el año 1610 por Pablo $V$ ésta recibe ya su forma definitiva de 20 capítulos que, a partir de entonces, se repetirá sin modificaciones.

Su entrada en España estuvo llena de dificultades desde el primer momento, por chocar contra las regalías de la Corona, especialmente desde Carlos V. Pero fue con Felipe II cuando las dificultades subieron de tono ante las quejas de los diversos Consejos Reales, por lo que el rey protestó formalmente ante el Papa Pío V y Gregorio XIII, y a partir de 1571 en Aragón parece que no volvió a publicarse. De hecho se llegó a la situación de que se admitía, salvo en aquellos capítulos que se estimaban lesivos para las regalías de la Corona. En diversas diócesis se editaba junto con las Constituciones sinodiales. Navarro, Toledo, Suárez y otros juristas se ocuparon de ella. Por fin, en 1770 se consiguió que Clemente XIV no la publicase más, aunque las censuras siguieron en vigor hasta 1869. De aquí que la polémica sobre la Bula fuera especialmente viva en los años en que redactó Campomanes este escrito. 